\title{
SÍNTESE, AVALIAÇÃo BIOLÓGICA E MODELAGEM MOLECULAR DE ARILFURANOS COMO INIBIDORES DA ENZIMA TRIPANOTIONA REDUTASE
}

Renata B. de Oliveira*\#, Carlos L. Zani, Rafaela S. Ferreira, Rodrigo S. Leite e Tânia M. A. Alves

Laboratório de Química de Produtos Naturais, Centro de Pesquisas René Rachou, Fiocruz, Av. Augusto de Lima, 1715, 30190-002 Belo Horizonte - MG, Brasil

Thaís H. A. da Silva

Departamento de Produtos Farmacêuticos, Faculdade de Farmácia, Universidade Federal de Minas Gerais, Av. Presidente Antônio Carlos, 6627, 31270-901 Belo Horizonte - MG, Brasil

\section{Alvaro J. Romanha}

Laboratório de Parasitologia Celular e Molecular, Centro de Pesquisas René Rachou, Fiocruz, Av. Augusto de Lima, 1715, 30190-002 Belo Horizonte - MG, Brasil

Recebido em 4/1/07; aceito em 13/7/07; publicado na web em 19/12/07

\begin{abstract}
SYNTHESIS, BIOLOGICAL EVALUATION AND MOLECULAR MODELING OF ARYLFURANS AS POTENTIAL TRYPANOTHIONE REDUCTASE INHIBITORS. Trypanosoma cruzi is a protozoan parasite that causes a severe disease (Chagas'disease) in Central and South America. The currently available chemotherapeutic agents against this disease are still inadequate. The enzyme trypanothione reductase (TR) is considered a validated molecular target for the development of new drugs against this parasite. In this regard, a series of arylfurans based on 2,5-bis-(4-acetamidophenyl)furan was synthesized and tested for their in vitro inhibitory activity against TR. Molecular modeling studies of putative enzyme-inhibitor complexes revealed a possible mechanism of interaction. From synthesized compounds, a benzylaminofuran derivative was found to be more active than the lead compound.
\end{abstract}

Keywords: trypanothione reductase; molecular modeling; arylfurans.

\section{INTRODUÇÃOO}

A doença de Chagas é uma doença tropical causada pelo hemoflagelado Trypanosoma cruzi. Segundo a Organização Mundial de Saúde, esta endemia atinge entre 16 a 18 milhões de pessoas distribuídas entre México, América Central e América do Sul. Estima-se que 13.000 pessoas morrem por ano vítimas dessa doença ${ }^{1}$.

Atualmente, o único fármaco disponível para o tratamento da Doença de Chagas é o benzonidazol, o qual é eficaz apenas na fase aguda da doença, sendo praticamente inócuo para o parasita na fase crônica, estágio em que se encontra a maioria dos chagásicos ${ }^{2}$. Além disso, esse fármaco causa reações adversas muitas vezes graves, entre as quais se destacam: dermatite por hipersensibilidade, depressão da medula óssea e polineuropatia periférica ${ }^{3}$. Portanto, existe uma necessidade urgente de novos agentes quimioterápicos para esta doença, que sejam mais eficazes e com menos efeitos colaterais.

Neste sentido, a descoberta de alvos que possam ser explorados no desenvolvimento de novos fármacos tripanosomicidas é de extrema importância. A enzima tripanotiona redutase (TR) foi descoberta em $1985^{4}$ e atualmente é considerada um alvo molecular validado para o planejamento de inibidores a serem utilizados no tratamento da doença de Chagas ${ }^{5,6}$. Esta enzima é dependente de NADPH e catalisa a redução da tripanotiona disulfeto $\left[\mathrm{T}(\mathrm{S})_{2}\right]$ em tripanotiona ditiol $\left[\mathrm{T}(\mathrm{SH})_{2}\right]$, desencadeando, assim, uma cascata de eventos responsáveis pela neutralização de espécies reativas de oxigênio (Figura 1). Desta forma, a TR mantém um ambiente redutor no interior do

*e-mail: renatabo@farmacia.ufmg.br

\#Endereço atual: Departamento de Produtos Farmacêuticos, Faculdade de Farmácia, Universidade Federal de Minas Gerais, Av. Presidente Antônio Carlos, 6627, 31270-901 Belo Horizonte - MG, Brasil

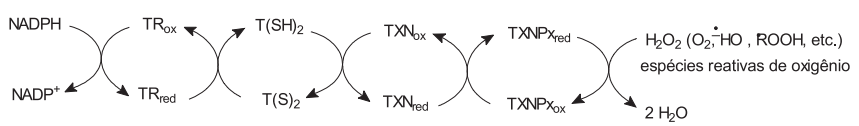

$\mathrm{TR}=$ tripanotiona redutase

TXN = triparedoxina
TXNPX = triparedoxina peroxidase

Figura 1. Cascata de eventos, dependentes da TR, responsáveis pela neutralização das espécies reativas de oxigênio

parasita, protegendo-o contra o estresse oxidativo ${ }^{5}$.

Além de exercer uma função fundamental para o parasita, uma outra característica que faz com que a TR seja um alvo interessante para o planejamento de fármacos antiparasitários é a sua significativa diferença estrutural em relação a glutationa redutase (GR), enzima com função correspondente no homem. As principais diferenças entre a TR e a GR estão relacionadas ao tamanho, carga e distribuição dos bolsões hidrofílicos/hidrofóbicos. Estas diferenças fazem com que o sítio ativo da TR, em comparação com o da GR, seja capaz de acomodar facilmente substratos apresentando grupos mais volumosos ${ }^{7}$. Além disso, a TR apresenta resíduos carregados negativamente e regiões hidrofóbicas em seu sítio ativo que são capazes de interagir, via interações eletrostáticas e de van der Waals, respectivamente, com seu substrato. Ao contrário, a GR apresenta resíduos de arginina, carregados positivamente, que interagem por ligação eletrostática com seu substrato ${ }^{8}$ (Figura 2). Estas diferenças podem ser aproveitadas no planejamento de inibidores seletivos para a TR.

Recentemente demonstramos que o 2,5-bis-(4-acetilaminofenil)furano (1) é capaz de inibir, na concentração de $48 \mu \mathrm{M}$, $50 \%$ da atividade da TR ${ }^{9}$. Apesar de apresentar uma atividade considerável como inibidor da TR, a diacetamida (1) não foi ativa nos 


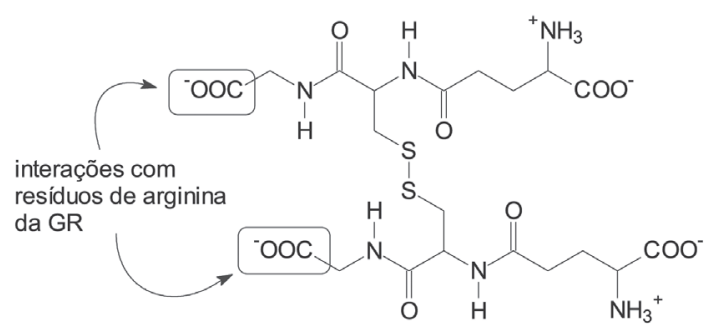

Glutationa

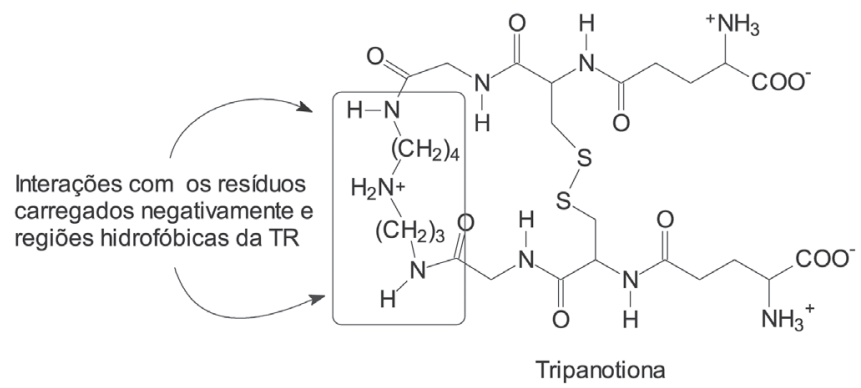

Figura 2. Comparação entre as estruturas da glutationa e tripanotiona, substratos da GR e TR, respectivamente

ensaios in vitro utilizando as formas tripomastigota e amastigota do T. cruzi. Uma explicação para este fato pode estar relacionada com a baixa solubilidade de $(\mathbf{1})(\operatorname{LogP}=0,39$, calculado usando o programa HyperChem 6.02), o que dificulta sua penetração no parasita e, conseqüentemente, a impede de alcançar concentrações suficientes para a inibição persistente da $\mathrm{TR}^{9}$.

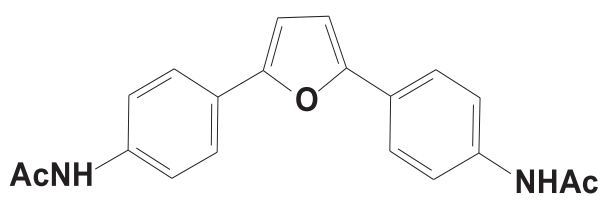

(1)

Visando a obtenção de inibidores mais ativos e que apresentem melhores características físico-químicas, uma série de aminas e amidas análogas a (1) foi planejada e sintetizada. Esperava-se que a associação de grupos capazes de formar de ligação de hidrogênio (-NH, -CHO, C=S, C=O, -NHCO) e grupos hidrofóbicos, pudesse aumentar a afinidade substrato-enzima. Sabendo-se que substâncias contendo grupos passíveis de sofrerem biorredução e gerarem radicais livres podem atuar como substratos subversivos para a $\mathrm{TR}^{6,10-13}$, planejou-se, também, a síntese de alguns análogos contendo grupo nitro em sua estrutura. As estruturas dos arilfuranos sintetizados neste trabalho são mostradas na Figura 3. Paralelamente, os compostos sintetizados foram submetidos a estudos de super-posição molecular utilizando o programa AutoDock 3.0 ${ }^{14}$, para dar subsídios ao entendimento sobre o modo de interação destes compostos com a enzima e, conseqüentemente, tornar possível o planejamento de novos inibidores.

\section{PARTE EXPERIMENTAL}

\section{Procedimentos gerais}

As temperaturas de fusão foram determinadas em aparelho Electrothermal IA9000 e não foram corrigidas. Os espectros de

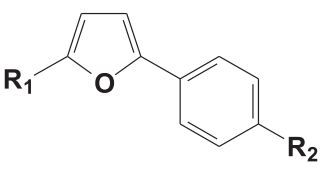

2-13

(2) $\mathrm{R}_{1}=\mathrm{H} ; \mathrm{R}_{2}=\mathrm{NH}_{2}$

(3) $\mathrm{R}_{1}=\mathrm{H} ; \mathrm{R}_{2}=\mathrm{NHAC}$

(4) $\mathrm{R}_{1}=\mathrm{H} ; \mathrm{R}_{2}=\mathrm{CONH}_{2}$

(5) $\mathrm{R}_{1}=\mathrm{H} ; \mathrm{R}_{2}=\mathrm{CH}_{2} \mathrm{NH}_{2}$

(6) $\mathrm{R}_{1}=\mathrm{H} ; \mathrm{R}_{2}=\mathrm{CH}_{2} \mathrm{NHAC}$

(7) $\mathrm{R}_{1}=\mathrm{CHO} ; \mathrm{R}_{2}=\mathrm{NO}_{2}$

(8) $\mathrm{R}_{1}=\mathrm{CHO} ; \mathrm{R}_{2}=\mathrm{NH}_{2}$

(9) $\mathrm{R}_{1}=\mathrm{CHO} ; \mathrm{R}_{2}=\mathrm{NHAC}$

(10) $\mathrm{R}_{1}=\mathrm{CHNNHCONH} \mathrm{H}_{2} ; \mathrm{R}_{2}=\mathrm{NO}_{2}$

(11) $\mathrm{R}_{1}=\mathrm{CHNNHCSNH} \mathrm{C}_{2} ; \mathrm{R}_{2}=\mathrm{NO}_{2}$

(12) $\mathrm{R}_{1}=\mathrm{CHNNHCONH} \mathrm{C}_{2} ; \mathrm{R}_{2}=\mathrm{NHAC}$

(13) $\mathrm{R}_{1}=\mathrm{CHNNHCSNH}_{2} ; \mathrm{R}_{2}=\mathrm{NHAC}$

Figura 3. Estruturas dos arilfuranos sintetizados

RMN de ${ }^{1} \mathrm{H}$ e de ${ }^{13} \mathrm{C}$ foram registrados no espectrômetro Bruker Avance DPX200, utilizando-se $\mathrm{CDCl}_{3}$ ou DMSO-d $\mathrm{d}^{6}$ como solventes e TMS como referência interna. Os espectros de massas foram obtidos em espectrômetro de massas modelo LCQ Advantage com armadilha de íon (Thermo Electron, San Jose, CA) equipado com fonte IES e operado no modo positivo. Os dados espectrais representam a média de 50 varreduras $(0,2 \mathrm{~s} /$ varredura $)$. As amostras foram inseridas por infusão utilizando seringa e bomba em um fluxo de $5 \mu \mathrm{L} \mathrm{min}{ }^{-1}$. As condições de IES foram: temperatura do capilar de $150{ }^{\circ} \mathrm{C}$; gás de arraste $\mathrm{N}_{2}$ com fluxo de 20 unidades; a voltagem do capilar foi de $4,5 \mathrm{kV}$.

Os reagentes, todos de grau analítico, foram utilizados sem purificação prévia. As cromatografias em coluna foram realizadas com sílica gel 60, 70-230 mesh. (Merck). A síntese e caracterização estrutural dos arilfuranos 17-19 já foram previamente descritas 9 .

\section{Sínteses}

\section{5-(4-nitrofenil)furano-2-carbaldeído $(7)^{15}$}

Em um balão de fundo redondo, imerso em um banho de gelo e sal, foram adicionados $0,7 \mathrm{~g}(5,1 \mathrm{mmol})$ da 4-nitroanilina, $3 \mathrm{~mL}$ de ácido clorídrico P.A. e $10 \mathrm{~mL}$ de água. Em seguida, adicionou-se, gota a gota, uma solução de 0,350 g (5,1 mmol) $\mathrm{NaNO}_{2}$ em $10 \mathrm{~mL}$ de água gelada. Após 5 min sob agitação magnética à temperatura de $5{ }^{\circ} \mathrm{C}$, adicionaram-se $0,5 \mathrm{~mL}(0,58 \mathrm{~g} ; 6,0 \mathrm{mmol})$ do furfural, 20 $\mathrm{mL}$ de acetona e $0,13 \mathrm{~g} \mathrm{de} \mathrm{CuCl}_{2} \cdot 2 \mathrm{H}_{2} \mathrm{O}(0,76 \mathrm{mmol})$. A reação foi mantida sob agitação magnética a temperatura ambiente durante 24 h. Após o término da reação, adicionou-se água destilada e filtrou-se o precipitado formado, lavando-se com água destilada. $\mathrm{O}$ sólido obtido foi purificado por cromatografia em coluna de sílica e o produto foi eluído com hexano/acetato de etila. Foi obtido 0,83 g (3,8 mmol) de 7, o que corresponde a 75\% de rendimento. Ponto de fusão: 203,3-203,9 ${ }^{\circ} \mathrm{C}$ (lit $\left.{ }^{15} .203{ }^{\circ} \mathrm{C}\right)$. RMN de ${ }^{1} \mathrm{H}(200$ MHz, DMSO-d 6 ): $\delta: 9,7$ (s, $1 \mathrm{H}, \mathrm{CHO}$ ), 8,35 (d. $2 \mathrm{H}, J=8,9 \mathrm{~Hz}$, H-aromáticos), 8,13 (d. 2H, $J=8,9 \mathrm{~Hz}, \mathrm{H}$-aromáticos), 7,72 (d, $1 \mathrm{H}, J=3,8 \mathrm{~Hz}, \mathrm{H}$-furano), 7,59 (d, $1 \mathrm{H}, J=3,8 \mathrm{~Hz}, \mathrm{H}$-furano).

\section{Metodologia geral: reação de redução do grupo nitro}

Em um balão de fundo redondo adicionaram-se o derivado nitrado 7 ou 17, metanol anidro e, lentamente, aproximadamente $10 \mathrm{mg}$ de carvão contendo $10 \%$ de Pd. O balão foi fechado com uma rolha de saia e eliminou-se o oxigênio do sistema com fluxo 
de nitrogênio. Em seguida, manteve-se a mistura reagindo sob atmosfera de hidrogênio durante $20 \mathrm{~h}$. Filtrou-se e evaporou-se o filtrado sob pressão reduzida. $\mathrm{O}$ produto obtido foi utilizado na próxima etapa de síntese, sem purificação prévia.

\section{5-(4-aminofenil)furano $(2)^{16}$}

A reação foi realizada conforme a metodologia geral para redução do grupo nitro, descrita acima. Foi obtido $0,93 \mathrm{~g}(5,8 \mathrm{mmol})$ de 2, o que corresponde a $100 \%$ de rendimento. Ponto de fusão: $50,7-53,5^{\circ} \mathrm{C}$. RMN de ${ }^{1} \mathrm{H}$ (200 MHz, DMSO-d $\left.{ }^{6}\right): \delta: 7,56\left(1 \mathrm{H}, \mathrm{dd}, J_{5,4}=1,8 \mathrm{~Hz}, \mathrm{H}-5\right.$, furano); 7,40 (2H, d, $J=8,6 \mathrm{~Hz}, \mathrm{H}$-aromáticos); 6,69 (2H, d, $J=8,6$ $\mathrm{Hz}, \mathrm{H}$-aromáticos); $6,56\left(1 \mathrm{H}, \mathrm{dd}, J_{3,4}=3,3, \mathrm{H}-3\right.$, furano); 6,47 (1H, dd, $J_{4,5}=1,8 \mathrm{~Hz}, J_{4,3}=3.3 \mathrm{~Hz} \mathrm{H}-4$, furano); 3,54 (2H, sl, $\left.\mathrm{NH}_{2}\right)$.

\section{5-(4-aminofenil)furano-2-carbaldeído $(8)^{17}$}

A reação foi realizada conforme a metodologia geral para redução do grupo nitro, descrita acima. Foi obtido $0,06 \mathrm{~g}(0,3 \mathrm{mmol})$ de $\mathbf{8}$, o que corresponde a $72 \%$ de rendimento. O produto obtido foi utilizado na próxima etapa de síntese, sem purificação prévia e não foi caracterizado.

\section{Metodologia geral: reação de redução da nitrila ${ }^{18}$}

Em um balão de fundo redondo contendo hidreto de lítio e alumínio, acoplou-se um funil de adição e adicionou-se, gota a gota, tetraidrofurano (THF) anidro, sob banho de gelo e fluxo contínuo de nitrogênio. Em seguida, adicionou-se, gota a gota, uma solução da nitrila em THF anidro. Deixou-se a mistura sob agitação magnética à temperatura ambiente e fluxo de nitrogênio durante $24 \mathrm{~h}$. Posteriormente, adicionaram-se à mistura, lentamente, $0,5 \mathrm{~mL}$ de água e 0,5 $\mathrm{mL}$ de solução de $\mathrm{NaOH}$ a 20\%. Filtrou-se e extraiu-se com 3 x $40 \mathrm{~mL}$ de éter etílico. Secou-se a fase orgânica com sulfato de sódio, filtrouse e evaporou-se o filtrado sob pressão reduzida. O produto obtido foi utilizado na próxima etapa de síntese, sem purificação prévia.

\section{2-(4-aminobenzil)furano (5)}

A reação foi realizada conforme a metodologia geral para redução de nitrila, descrita acima. Foi obtido $0,14 \mathrm{~g}(0,81 \mathrm{mmol})$ de 5, o que corresponde a $69 \%$ de rendimento. P.F.: $98,3-99,9{ }^{\circ} \mathrm{C}$; RMN de $\left.{ }^{1} \mathrm{H}(200 \mathrm{MHz}, \text { DMSO-d })^{6}\right):$ : 7,72-7,62 (4H, m, 4xH-aromáti$\cos ) ; 7,39\left(1 \mathrm{H}, \mathrm{d}, J_{5,4}=1,8, \mathrm{H}-5\right.$ furano $) ; 6,90\left(1 \mathrm{H}, \mathrm{d}, J_{3,4}=3,3, \mathrm{H}-\right.$ 3 furano); $6,58\left(1 \mathrm{H}, \mathrm{dd}, J_{4,5}=1,8 \mathrm{~Hz}, J_{4,3}=3,3 \mathrm{~Hz}, \mathrm{H}-4\right.$ furano); 3,75-3,50 (4H, m, 2xH-benzílicos e $\left.\mathrm{NH}_{2}\right)$. IES-EM: $[\mathrm{M}+\mathrm{H}]^{+} 174$.

\section{2,5-bis-(4-aminobenzil)furano $(\mathbf{1 5})^{19}$}

A reação foi realizada conforme a metodologia geral para redução de nitrila, descrita acima. Foi obtido $0,38 \mathrm{~g}(1,37 \mathrm{mmol})$ de $\mathbf{1 5}$, o que corresponde a $76 \%$ de rendimento. P.F.: $108,4-110,3{ }^{\circ} \mathrm{C}$. RMN de ${ }^{1} \mathrm{H}\left(200 \mathrm{MHz}, \mathrm{DMSO}-\mathrm{d}^{6}\right): \delta: 7,73(4 \mathrm{H}, \mathrm{d}, J=7,9 \mathrm{~Hz}, 4 \times \mathrm{H}$-aromáticos); 7,40 (4H, d, $J=7,9 \mathrm{~Hz}, 4 \times \mathrm{H}$-aromáticos); 6,99 (2H, s, H-3 e H-4 furano); 3,74-3,69 (8H, m, 4xH-benzílicos e 2xNH${ }_{2}$ ).

\section{Metodologia geral: reação de acetilação do grupo amino ${ }^{20}$}

Em um balão de fundo redondo contendo o derivado amino, adicionaram-se anidrido acético e uma solução de acetato de sódio a $5 \%$. Deixou-se a mistura sob agitação magnética e banho de gelo por cerca de $30 \mathrm{~min}$. Adicionou-se, então, água destilada e transferiu-se a mistura para um funil de separação. Extraiu-se com 3 x 40 $\mathrm{mL}$ de acetato de etila. Secou-se a fase orgânica com sulfato de sódio, filtrou-se e evaporou-se o filtrado sob pressão reduzida. O resíduo obtido foi purificado por cromatografia em coluna, utilizando hexano/acetato de etila como eluentes.

\section{2-(4-acetilaminofenil)furano $(\mathbf{3})^{\mathbf{2 1}}$}

A reação foi realizada conforme a metodologia geral para acetilação de grupo amino, descrita acima. Foi obtido $0,44 \mathrm{~g}(2,19$ mmol) de 3, o que corresponde a $50 \%$ de rendimento. Ponto de fusão: $185,4-187,7{ }^{\circ} \mathrm{C}$. RMN de ${ }^{1} \mathrm{H}\left(200 \mathrm{MHz}, \mathrm{CDCl}_{3}\right): \delta: 10,04$ $(1 \mathrm{H}, \mathrm{sl}, \mathrm{NH}) ; 7,68\left(1 \mathrm{H}, \mathrm{d}, J_{54}=1,8 \mathrm{~Hz}, \mathrm{H}-5\right.$ furano $) ; 7,63(4 \mathrm{H}, \mathrm{m}$, H-aromáticos); 6,81 (1H, d, $J_{3,4}=3,2 \mathrm{~Hz}, \mathrm{H}-3$ furano); 6,55 (1H,dd, $J_{4,5}=1,8 \mathrm{~Hz} ; J_{4,3}=3,2 \mathrm{~Hz}, \mathrm{H}-4$ furano).

\section{5-(4-acetilaminofenil)furano-2-carbaldeído $(\mathbf{9})^{17}$}

A reação foi realizada conforme a metodologia geral para acetilação de grupo amino, descrita acima. Foi obtido 0,014 g (0,06 mmol) de 9, o que corresponde a $23 \%$ de rendimento. P.F.: 181,7$185,4{ }^{\circ} \mathrm{C}$; RMN de ${ }^{1} \mathrm{H}\left(200 \mathrm{MHz}, \mathrm{DMSO}-\mathrm{d}^{6}\right): \delta: 10,19$ (1H, s, CHO); 9,57 (1H, s, NH), 7,82 (2H, d, $J=8,9 \mathrm{~Hz}, \mathrm{H}$-aromáticos), 7,72 (2H, d, $J=8,9 \mathrm{~Hz}, \mathrm{H}$-aromáticos), 7,64 $\left(1 \mathrm{H}, \mathrm{d}, J_{3,4}=3,8 \mathrm{~Hz}, \mathrm{H}-3\right.$ furano); $7,17\left(1 \mathrm{H}, \mathrm{d}, J_{4,3}=3,8 \mathrm{~Hz}, \mathrm{H}-4\right.$ furano), 2,08 (3H, s, $\left.\mathrm{CH}_{3} \mathrm{CO}\right)$.

\section{2-(4-acetilaminobenzil)furano (6)}

A reação foi realizada conforme a metodologia geral para acetilação de grupo amino, descrita acima. Foi obtido 0,063 g (0,29 mmol) de 6, o que corresponde a $51 \%$ de rendimento. P.F. $=145,3-$ $147,4{ }^{\circ} \mathrm{C}$; RMN de ${ }^{1} \mathrm{H}\left(200 \mathrm{MHz}, \mathrm{DMSO}-\mathrm{d}^{6}\right): \delta: 8,38(1 \mathrm{H}, \mathrm{t}, J=5,9$ $\mathrm{Hz}, \mathrm{NH}), 7,73\left(1 \mathrm{H}, \mathrm{d}, J_{5,4}=1,4 \mathrm{~Hz}, \mathrm{H}-5\right.$ furano), 7,65 $(2 \mathrm{H}, \mathrm{d}, J=$ $8,2 \mathrm{~Hz}, 2 \mathrm{xH}$-aromáticos $), 7,30(2 \mathrm{H}, \mathrm{d}, J=8,2 \mathrm{~Hz}, 2 \mathrm{xH}$-aromáti$\cos ) ; 6,91\left(1 \mathrm{H}, \mathrm{d}, J_{3,4}=3,3 \mathrm{~Hz}, \mathrm{H}-3\right.$ furano), 6,58 ( $1 \mathrm{H}, \mathrm{dd}, J_{4,5}=1,4$ $\mathrm{Hz}, J_{4,3}=3,3 \mathrm{~Hz}, \mathrm{H}-4$ furano $), 4,27(2 \mathrm{H}, \mathrm{d}, J=5,9 \mathrm{~Hz}, 2 \mathrm{xH}-$ benzílicos), 1,89 (3H, s, $\left.\mathrm{CH}_{3} \mathrm{CO}\right)$; IES-EM: $[\mathrm{M}+\mathrm{H}]^{+}$216, $[\mathrm{M}+\mathrm{Na}]^{+}$ $238,[2 \mathrm{M}+\mathrm{H}]^{+} 431,[2 \mathrm{M}+\mathrm{Na}]^{+} 453$.

\section{2,5-bis-(4-acetilaminobenzil)furano (16)}

A reação foi realizada conforme a metodologia geral para acetilação de grupo amino, descrita acima. Foi obtido $0,23 \mathrm{~g}(0,64$ mmol) de 16, o que corresponde a $58 \%$ de rendimento. P.F.: 263,4266,7 ${ }^{\circ} \mathrm{C}$; RMN de ${ }^{1} \mathrm{H}\left(200 \mathrm{MHz}, \mathrm{DMSO}-\mathrm{d}^{6}\right): \delta: 7,71(2 \mathrm{H}, \mathrm{t}, J=5,8$ $\mathrm{Hz}, 2 \mathrm{xNH}), 7,08(4 \mathrm{H}, \mathrm{d}, J=8,1 \mathrm{~Hz}, 4 \mathrm{xH}$-aromáticos), 6,65 (4H, d, $J=8,1 \mathrm{~Hz}, 4 \mathrm{xH}$-aromáticos), 6,36 (2H, s, H-3 e H-4 furano); 3,59

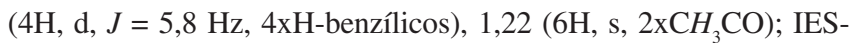
EM: $[\mathrm{M}+\mathrm{Na}]^{+} 385,[2 \mathrm{M}+\mathrm{Na}]^{+} 747$.

\section{2-(4-benzamida)furano (4)}

Em um balão de fundo redondo adicionaram-se $352 \mathrm{mg}$ de $\mathbf{1 8}$ (2,1 mmol), $10 \mathrm{~mL}$ de etanol e $10 \mathrm{~mL}$ de dioxano. Em seguida, adicionaram-se uma solução de 2,5 g de hidróxido de sódio (62,5 mmol) em $10 \mathrm{~mL}$ de água e $15 \mathrm{~mL}$ de $\mathrm{H}_{2} \mathrm{O}_{2} 20 \%$. A mistura foi aquecida a $60{ }^{\circ} \mathrm{C}$ durante $2 \mathrm{~h}$. A mistura foi extraída com 3 × $50 \mathrm{~mL}$ de acetato de etila. As fases orgânicas foram reunidas, adicionouse sulfato de sódio anidro e filtrou-se. O filtrado foi, então, evaporado sob pressão reduzida e o sólido obtido foi purificado por recristalização em etanol ${ }^{22}$. Foi obtido $0,140 \mathrm{~g}(0,75 \mathrm{mmol})$ de 4, o que corresponde a $36 \%$ de rendimento. P.F.: $232,7-235,0{ }^{\circ} \mathrm{C}$; RMN de ${ }^{1} \mathrm{H}\left(200 \mathrm{MHz}, \mathrm{DMSO}-\mathrm{d}^{6}\right): \delta 8,02(1 \mathrm{H}, \mathrm{sl}, \mathrm{N} H) ; 7,94(2 \mathrm{H}, \mathrm{d}, J=$ $8,5 \mathrm{~Hz}, \mathrm{H}$-aromáticos); 7,80 (1H, d, $J_{5,4}=1,8 \mathrm{~Hz}, \mathrm{H}-5$ furano); 7,78 $(2 \mathrm{H}, \mathrm{d}, J=8,5 \mathrm{~Hz}, \mathrm{H}$-aromáticos); 7,40 (1H, sl, NH); 7,10 (1H, d, $J_{3,4}=3,4 \mathrm{~Hz}, \mathrm{H}-3$ furano); $6,64\left(1 \mathrm{H}, \mathrm{dd}, J_{4,5}=1,8 \mathrm{~Hz} . J_{4,3}=3,4 \mathrm{~Hz}\right.$, $\mathrm{H}-4$ furano); IES-EM: $[\mathrm{M}+\mathrm{H}]^{+} 188$.

\section{2,5-bis-(4-benzamida)furano (14)}

Aqueceu-se a $85^{\circ} \mathrm{C}, 157 \mathrm{mg}$ de $19(0,58 \mathrm{mmol})$ em $1 \mathrm{~mL}$ de ácido sulfúrico concentrado, sob agitação magnética, durante $5 \mathrm{~min}$. A solução foi, então, resfriada e lentamente vertida sobre $5 \mathrm{~mL}$ de água gelada, resultando na formação de um precipitado. O precipitado foi separado por filtração a vácuo, agitado com $5 \mathrm{~mL}$ de solução de hidróxido 
de sódio $5 \%$ e filtrado novamente $\mathrm{e}^{20}$. Foi obtido $0,08 \mathrm{~g}(0,26 \mathrm{mmol}) \mathrm{de}$ 14, o que corresponde a $44 \%$ de rendimento. $\mathrm{RMN}$ de ${ }^{1} \mathrm{H}(200 \mathrm{MHz}$, DMSO-d $\left.{ }^{6}\right): \delta 8,09$ (2H, sl, 2xN-H); 7,96 (8H, m, 8xH-aromáticos); 7,44 (2H, sl, 2xN-H); 7,27 (2H, s, H-4 e H-3 furano).

\section{Metodologia geral: síntese das semicarbazonas}

Em um balão de fundo redondo contendo uma solução do aldeído (1 equivalente) em etanol, adicionaram-se uma solução do cloridrato de semicarbazida (1 equivalente) e acetato de sódio (1 equivalente) em água. A mistura foi aquecida a $40{ }^{\circ} \mathrm{C}$ durante $20 \mathrm{~h}$. Adicionou-se água destilada e a mistura foi transferida para um funil de separação e extraiu-se com 3 x $30 \mathrm{~mL}$ de acetato de etila. Secou-se a fase orgânica com sulfato de sódio, filtrou-se e evaporou-se o filtrado sob pressão reduzida. O resíduo obtido foi purificado por CCS utilizando hexano/acetato de etila como eluentes.

\section{5-(4-nitrofenil)-2-furfurilideno semicarbazona $(\mathbf{1 0})^{23}$}

A reação foi realizada conforme a metodologia geral para síntese de semicarbazona, descrita acima. Foi obtido 0,025 g (0,09 mmol) de 10, o que corresponde a $38 \%$ de rendimento. P.F.: $210,8-$ 212,8 ${ }^{\circ} \mathrm{C}$; RMN de ${ }^{1} \mathrm{H}\left(200 \mathrm{MHz}, \mathrm{DMSO}-\mathrm{d}^{6}\right): \delta 10,46(1 \mathrm{H}, \mathrm{s}, \mathrm{CHO})$; $8,26(2 \mathrm{H}, \mathrm{d}, J=8,9 \mathrm{~Hz}, \mathrm{H}$-aromáticos $) ; 8,02(2 \mathrm{H}, \mathrm{d}, J=8,9 \mathrm{~Hz}, \mathrm{H}-$ aromáticos); 7,80 (1H, s, NH); 7,40 $\left(1 \mathrm{H}, \mathrm{d}, J_{3,4}=3,6 \mathrm{~Hz}, \mathrm{H}-3\right.$ furano); $7,00\left(1 \mathrm{H}, \mathrm{d}, J_{4,3}=3,6 \mathrm{~Hz}, \mathrm{H}-4\right.$ furano); 6,52 $\left(2 \mathrm{H}, \mathrm{s}, \mathrm{NH}_{2}\right)$.

\section{5-(4-acetilaminofenil)-2-furfurilideno semicarbazona (12)}

A reação foi realizada conforme a metodologia geral para síntese de semicarbazona, descrita acima. Foi obtido 0,050 g $(0,17$ mmol) de 12, o que corresponde a $79 \%$ de rendimento. P.F.: $244,1-$ $247,0{ }^{\circ} \mathrm{C}$; RMN de ${ }^{1} \mathrm{H}\left(200 \mathrm{MHz}, \mathrm{DMSO}-\mathrm{d}^{6}\right): \delta 10,29(1 \mathrm{H}, \mathrm{s}, \mathrm{CHO}$ ou $\mathrm{NH}), 10,07(1 \mathrm{H}, \mathrm{s}, \mathrm{NH}$ ou $\mathrm{CHO}), 7,72(2 \mathrm{H}, \mathrm{d}, J=8,9 \mathrm{~Hz}, 2 \mathrm{xH}-$ aromáticos); 7,64 $(2 \mathrm{H}, \mathrm{d}, J=8,9 \mathrm{~Hz}, 2 \times \mathrm{H}$-aromáticos), 6,93 $(1 \mathrm{H}$, $\mathrm{d}, J_{34}=3,4 \mathrm{~Hz}, \mathrm{H}-3$ furano), 6,87 $\left(1 \mathrm{H}, \mathrm{d}, J_{43}=3,4 \mathrm{~Hz}, \mathrm{H}-4\right.$ furano), 6,42 $\left(2 \mathrm{H}, \mathrm{s}, \mathrm{NH}_{2}\right), 2,05\left(3 \mathrm{H}, \mathrm{s}, \mathrm{CH}_{3} \mathrm{CO}\right)$. IES-EM: $[\mathrm{M}+\mathrm{H}]^{+} 287$.

\section{Metodologia geral: síntese das tiossemicarbazonas}

Em um balão de fundo redondo contendo uma solução do aldeído (1 equivalente) em etanol e ácido acético, adicionou-se uma solução da tiossemicarbazida (1 equivalente) em etanol. A mistura foi aquecida a $40{ }^{\circ} \mathrm{C}$ durante $20 \mathrm{~h}$. Adicionou-se água destilada e a mistura foi transferida para um funil de separação e extraiu-se com 3 x $30 \mathrm{~mL}$ de acetato de etila. Secou-se a fase orgânica com sulfato de sódio, filtrou-se e evaporou-se o filtrado sob pressão reduzida. O resíduo obtido foi purificado por CCS utilizando hexano/acetato de etila como eluentes.

\section{5-(4-nitrofenil)-2-furfurilideno tiossemicarbazona $(11)^{24}$}

A reação foi realizada conforme a metodologia geral para síntese de tiossemicarbazona, descrita acima. Foi obtido 0,042 g (0,14 mmol) de 11, o que corresponde a $38 \%$ de rendimento. P.F.: 211,2213,6 ${ }^{\circ} \mathrm{C}$; RMN de ${ }^{1} \mathrm{H}\left(200 \mathrm{MHz}, \mathrm{DMSO}-\mathrm{d}^{6}\right): \delta 11,62(1 \mathrm{H}, \mathrm{s}, \mathrm{CHO})$; $8,39(1 \mathrm{H}, \mathrm{s}, \mathrm{NH}) ; 8,24(2 \mathrm{H}, \mathrm{d}, J=8,9 \mathrm{~Hz}, 2 \mathrm{xH}$-aromáticos $) ; 8$,067,99 (3H, m, 2xH-aromáticos, $\mathrm{NH}) ; 7,87(1 \mathrm{H}, \mathrm{s}, \mathrm{NH}) ; 7,41(1 \mathrm{H}, \mathrm{d}$, $J_{3,4}=3,6 \mathrm{~Hz}, \mathrm{H}-3$ furano); 7,14 $\left(1 \mathrm{H}, \mathrm{d}, J_{4,3}=3,6 \mathrm{~Hz}, \mathrm{H}-4\right.$ furano).

\section{5-(4-acetilaminofenil)-2-furfurilideno tiossemicarbazona (13)}

A reação foi realizada conforme a metodologia geral para síntese de tiossemicarbazona, descrita acima. Foi obtido 0,026 g $(0,09$ mmol) de 13, o que corresponde a $39 \%$ de rendimento. P.F.: 191,6193,0 ${ }^{\circ} \mathrm{C}$; RMN de ${ }^{1} \mathrm{H}\left(200 \mathrm{MHz}, \mathrm{DMSO}-\mathrm{d}^{6}\right): \delta 11,47$ (1H, s, CHO), $10,08(1 \mathrm{H}, \mathrm{s}, \mathrm{NH}), 7,96\left(2 \mathrm{H}, \mathrm{s}, \mathrm{NH}_{2}\right), 7,76(2 \mathrm{H}, \mathrm{d}, J=8,7 \mathrm{~Hz}, 2 \mathrm{xH}-$ aromáticos); 7,65 (2H, d, $J=8,7 \mathrm{~Hz}, 2 x \mathrm{H}$-aromáticos), 7,03 (1H, $\mathrm{d}, J_{3,4}=3,5 \mathrm{~Hz}, \mathrm{H}-3$ furano), 6,97 $\left(1 \mathrm{H}, \mathrm{d}, J_{4,3}=3,5 \mathrm{~Hz}, \mathrm{H}-4\right.$ furano), $2,02\left(3 \mathrm{H}, \mathrm{s}, \mathrm{CH}_{3} \mathrm{CO}\right)$; IES-EM: $[\mathrm{M}+\mathrm{H}]^{+} 303$.

\section{Ensaios biológicos}

\section{Ensaio com a enzima tripanotiona redutase ${ }^{9}$}

O ensaio colorimétrico foi conduzido em placas de 96 poços sendo que cada poço $(250 \mu \mathrm{L})$ continha: $40 \mathrm{mM}$ de HEPES $(\mathrm{pH}$ 7,5), $1 \mathrm{mM}$ de EDTA, 5,12 mUI da enzima (TR), 0,8 $\mu \mathrm{M}$ do substrato $\left(\mathrm{T}(\mathrm{S})_{2}\right), 0,12 \mathrm{mM}$ de NADPH, $100 \mu \mathrm{M}$ da substância a ser testada (solução aquosa contendo $0,1 \%$ de DMSO). Controles com 6,5 $\mu \mathrm{M}$ de clomipramina (controle positivo) e sem amostra (controle negativo) foram conduzidos em paralelo. A mistura foi pré-encubada a $30{ }^{\circ} \mathrm{C}$ durante $30 \mathrm{~min}$ e a leitura iniciada após a adição de $25 \mu \mathrm{M}$ de DTNB (reagente de Ellman) em $\lambda_{410} \mathrm{~nm}$, medindo-se a inclinação da curva $(\delta \mathrm{Abs} / \delta \mathrm{t})$. Este valor foi comparado com o controle sem amostra para fornecer o percentual de inibição da enzima. Os ensaios foram realizados em triplicata e repetidos por, no mínimo, duas vezes. Foram consideradas ativas as amostras capazes de inibir em pelo menos $50 \%$ a atividade da enzima.

\section{Ensaio in vitro com T. cruzi $^{9}$}

Sangue de camundongos infectados com as formas tripomastigotas de cepas Y de T. cruzi foi utilizado nos experimentos. As substâncias foram testadas em placas de 96 poços, sendo que cada poço continha: $195 \mu \mathrm{L}$ de sangue infectado $\left(2,4 \times 10^{6}\right.$ parasitas $/ \mathrm{mL})$ e $5 \mu \mathrm{L}$ da solução da substância a ser testada em DMSO. As placas foram mantidas a $4{ }^{\circ} \mathrm{C}$. Após $24 \mathrm{~h}$, a concentração do parasita foi avaliada utilizando microscopia ótica. A redução da concentração do parasita (lise do parasita) foi determinada em comparação com os parasitas não tratados. Controle positivo $(7,5 \mu \mathrm{g} /$ $\mathrm{mL} ; 3,0 \mu \mathrm{M}$ de cristal violeta) e controle do solvente $(2,5 \%$ de DMSO v/v) foram realizados em todos os experimentos. Os ensaios foram realizados em duplicata e repetidos duas vezes.

\section{Estudos de superposição molecular}

As coordenadas da tripanotiona redutase complexada com a tripanotiona foram obtidas do "Brookhaven Protein Data Bank" (código PDB 1BZL). As moléculas de água e as moléculas de tripanotiona foram extraídas do complexo. As moléculas de FAD e a proteína foram submetidas separadamente a cálculos de cargas atômicas. As cargas foram determinadas utilizando métodos disponíveis no programa AutoDock 3.0 $0^{14}$. Para a macromolécula foram adicionados os hidrogênios polares (hidrogênios ligados a heteroátomos) e calculadas as cargas Kollman ${ }^{25}$. Ao resíduo de FAD foram adicionados todos os hidrogênios, calculadas as cargas Gasteiger ${ }^{26}$ e os hidrogênios não polares foram reunidos aos carbonos aos quais estavam ligados. Os arquivos da proteína e do FAD foram reunidos em um único arquivo para efetuar os cálculos de superposição. $\mathrm{O}$ AutoDock requer mapas de grade de afinidade pré-calculados para cada um dos tipos de átomos presentes nos ligantes a serem submetidos à superposição. Os mapas das grades de afinidade consistem de uma rede de pontos, espaçados regularmente, que armazenam a energia potencial de um átomo "prova" em relação a todos os átomos da macromolécula. As grades de afinidade foram então geradas usando o programam auxiliar AutoGrid ${ }^{27}$. Um dos átomos de enxofre da tripanotiona no sítio ativo foi escolhido como centro da grade que foi construída com $71 \times 71 \times 71$ pontos com espaçamento de 0,375 $\AA ̊$ entre os pontos da grade. Constatou-se que a macromolécula apresenta átomos de fósforo cujos parâmetos Lennard-Jones não esta- 
vam incluídos no programa AutoDock. Estes, então, foram defini$\operatorname{dos}\left(\mathrm{R}_{\mathrm{ij}}=4,2 \AA\right.$ e $\left.\mathrm{E}_{\mathrm{ij}}=0,2 \mathrm{kcal} \mathrm{mol}^{-1}\right)$ com base na literatura ${ }^{25}$.

Nos cálculos utilizando o AutoGrid e o AutoDock foram empregados potenciais para ligações de hidrogênio envolvendo os heteroátomos $\mathrm{O}, \mathrm{N}$ e $\mathrm{S}$, tanto para o ligante quanto para a macromolécula ${ }^{14}$. No experimento de superposição molecular foram realizadas 50 corridas de algoritmo genético Lamarckiano (algoritmo genético com busca local) e o número máximo de avaliações de energia de 500.000 e os outros parâmetros de algoritmo genético (GA) e busca local (LS) de acordo com o descrito por Morris e colaboradores ${ }^{14}$. A energia livre é utilizada no cálculo da constante de inibição $\mathrm{K}_{\mathrm{i}}$, usando a equação $\Delta \mathrm{G}=\mathrm{RT} \ln \mathrm{K}_{\mathrm{i}}$, onde $\mathrm{R}$ é a constante dos gases $\left(1,987 \mathrm{cal} \mathrm{K}^{-1} \mathrm{~mol}^{-1}\right)$ e T é a temperatura absoluta, assumida como temperatura ambiente, $298,15 \mathrm{~K}$. Observe que esta equação perdeu seu sinal negativo porque a constante de inibição é definida para a reação de dissociação, $\mathrm{E} \rightleftarrows \mathrm{E}+\mathrm{I}$, enquanto $\Delta \mathrm{G}_{\mathrm{obs}}$ refere-se ao processo oposto de ligação $E+\mathrm{I} \rightleftarrows E$, onde $E$ é a enzima e I o inibidor. Para facilitar a análise dos dados, os valores de $\mathrm{K}_{\mathrm{i}}$ foram transformados nos seus respectivos $\mathrm{pK}_{\mathrm{i}}\left(\mathrm{pK}_{\mathrm{i}}=-\log \mathrm{K}_{\mathrm{i}}\right)$.

Após a realização dos cálculos de superposição molecular e da variação de $\Delta \mathrm{G}$ envolvida na formação do complexo ligante-enzima, analisaram-se as interações químicas e o posicionamento da conformação energeticamente mais estável de cada ligante no sítio ativo da TR. Para tanto se construíram arquivos contendo a conformação mais estável de cada ligante, prevista pelo AutoDock, e todos os resíduos de aminoácidos próximos ao ligante. Foram identificados todos os heteroátomos da macromolécula a uma distância inferior a 3,5 ̊̊ de um heteroátomo do ligante, como possíveis pontos de formação de ligação de hidrogênio ${ }^{28}$.

\section{RESULTADOS E DISCUSSÃO}

\section{Síntese}

Os arilfuranos 7, 17-19 foram obtidos, utilizando o furano ou furfural e a 4-nitroanilina ou 4-aminobenzonitrila como materiais de partida ${ }^{9}$, de acordo com a metodologia de síntese descrita por Meerwein ${ }^{29}$.

Os nitroarilfuranos $\mathbf{7}$ e $\mathbf{1 7}$ foram submetidos à reação de hidrogenação catalítica, utilizando $\mathrm{H}_{2}$ sob $\mathrm{Pd} / \mathrm{C}$, resultando na obtenção dos derivados amino $\mathbf{2}$ e $\mathbf{8}$, respectivamente. Acetilação de $\mathbf{2}$ e 8 levou à formação das monoacetamidas $\mathbf{3}$ e $\mathbf{9}$, respectivamente.

A redução das nitrilas 18 e 19 utilizando $\mathrm{LiAlH}_{4}$ resultou na formação das benzilaminas $\mathbf{5}$ e $\mathbf{1 5}$ que foram, em seguida, submetidas à reação de acetilação, originando as benzilacetamidas 6 e 16, respectivamente.

A hidrólise das nitrilas $\mathbf{1 8}$ e $\mathbf{1 9}$ foi realizada, para fins de comparação, utilizando-se duas condições de reação: em meio básico, em um processo catalisado por $\mathrm{H}_{2} \mathrm{O}_{2}$ e, em meio ácido, por reação com ácido sulfúrico concentrado. No caso da nitrila $\mathbf{1 8}$, a hidrólise em meio ácido resultou na degradação do material de partida com a formação de produtos mais polares, difíceis de serem isolados, purificados e identificados. Entretanto, realizando-se a reação em meio básico, foi possível obter a monobenzamida 4 com $36 \%$ de rendimento. Os resultados obtidos na hidrólise da dinitrila 19 foram opostos aos da monotrila 18. A reação realizada em meio ácido apresentou melhor rendimento ( $44 \%$ de rendimento) que a reação em meio básico ( $10 \%$ de rendimento).

As semicarbazonas $\mathbf{1 0}$ e $\mathbf{1 2}$ foram obtidas reagindo-se os aldeídos correspondentes $\mathbf{7}$ e $\mathbf{9}$, respectivamente, com cloridrato de semicarbazida. Da mesma forma, as tiossemicarbazonas 11 e 13 foram obtidas por reação do aldeído correspondente com a tiossemicarbazida.
A obtenção de semicarbazonas e tiossemicarbazonas foi baseada no fato de que compostos contendo tais grupos, freqüentemente, apresentam um amplo perfil farmacológico e constituem uma importante classe de compostos cujas propriedades têm sido extensivamente estudadas em Química Medicinal ${ }^{30}$. Além disso, recentemente, foi demonstrado que 5-nitrofurfural-2-carboxaldeído semicarbazonas (Figura 4) são potenciais inibidores da TR e apresentam considerável atividade tripanosomicida ${ }^{10,31}$.

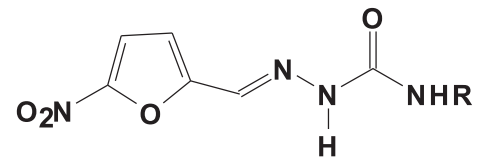

Figura 4. Estrutura geral de 5-nitrofurfural-2-carboxaldeído semicarbazonas

Pode-se observar que a semicarbazona $\mathbf{1 0}$ é um análago da 5nitrofurfural-2-carboxaldeído semicarbazona $(\mathrm{R}=\mathrm{H})$, sendo a única diferença a presença de um anel aromático entre o grupo nitro e o furano.

\section{Ensaio com a enzima tripanotiona redutase $X$ estudos de superposição molecular}

Todas as substâncias sintetizadas foram testadas, na concentração de $100 \mu \mathrm{M}$, no ensaio in vitro com a enzima TR. Controles com $6,5 \mu \mathrm{M}$ de clomipramina (controle positivo) e sem amostra (controle negativo) foram conduzidos em paralelo. Os ensaios foram realizados em triplicata e repetidos por, no mínimo, duas vezes.

Com o objetivo de testar a consistência do método computacional descrito na Parte Experimental, foi realizado um experimento de superposição da tripanotiona no sítio ativo da TR. A solução de menor energia calculada é muito similar à posição da tripanotiona revelada na estrutura cristalográfica do complexo TRtripanotiona depositado no PDB (1BZL). Foram observadas as seguintes interações da tripanotiona com a TR: um bolso de ligação com uma superfície hidrofóbica formada pelos resíduos LEU18, TRP22, TYR111, MET114, PHE396' e THR397' voltada para o lado onde se encontra a porção espermidina da tripanotiona, e um sítio hidrofílico composto de aminoácidos com distâncias adequadas para formação de ligações de hidrogênio com a porção tripeptídica da tripanotiona constituído dos seguintes resíduos: SER15, LEU18, GLU19, SER110, TYR111, PRO336, LEU399', HIS461', THR463', SER464', GLU466' e SER470'.

Confirmada a consistência do método, os experimentos utilizando os arilfuranos 1-16 como substratos para TR foram conduzidos de acordo com as condições estabelecidas anteriormente, originando os resultados mostrados na Tabela 1, onde também estão registrados os resultados dos ensaios de inibição da TR.

$\mathrm{O}$ valor de $\mathrm{K}_{\mathrm{i}}$ foi calculado diretamente a partir de $\Delta \mathrm{G}$, sendo menor para valores de $\Delta \mathrm{G}$ mais negativos. Em geral, teoricamente, os compostos que apresentaram maior porcentagem de inibição da enzima nos estudos in vitro deveriam apresentar os menores valores de $\Delta \mathrm{G}$ e, portanto, maiores valores de $\mathrm{pK}_{\mathrm{i}}$. A constante de inibição é a medida intrínseca da afinidade do inibidor pela enzima, enquanto outros fatores podem influenciar a medida da porcentagem de inibição ${ }^{32}$. A partir da análise dos resultados obtidos, verifica-se a ocorrência dessa relação em alguns dos casos. Os compostos com maior porcentagem de inibição (1 e 15) apresentaram os maiores valores de pKi e o de menor porcentagem de inibição (3) apresentou o menor valor de pKi.

Após a realização dos cálculos de energia de interação entre ligante e enzima, analisaram-se as interações químicas dos arilfuranos no sítio ativo da enzima, considerando em cada caso o 
Tabela 1. Porcentagem de inibição da enzima tripanotiona redutase e constantes de inibição previstas pelo programa AutoDock para os compostos sintetizados

\begin{tabular}{llll}
\hline Substrato & \multicolumn{2}{c}{ Dados Teóricos } & $\begin{array}{c}\text { Dados } \\
\text { Experimentais }\end{array}$ \\
\hline & $\Delta \mathrm{G}$ & $\mathrm{pK}$ & $\begin{array}{c}\% \text { inibição } \\
(100 \mu \mathrm{M})\end{array}$ \\
\hline Tripanotiona & $-12,77$ & 9,36 & - \\
$\mathbf{1}$ & $-8,6$ & 6,3 & $62,3 \pm 15,1$ \\
$\mathbf{2}$ & $-7,78$ & 5,7 & $7,0 \pm 3,5$ \\
$\mathbf{3}$ & $-5,85$ & 4,3 & $5,9 \pm 1,0$ \\
$\mathbf{4}$ & $-6,64$ & 4,9 & $32,6 \pm 0,7$ \\
$\mathbf{5}$ & $-7,52$ & 5,5 & $44,9 \pm 13,1$ \\
$\mathbf{6}$ & $-6,24$ & 4,6 & $24,2 \pm 4,1$ \\
$\mathbf{7}$ & $-7,75$ & 5,7 & $36,2 \pm 1,1$ \\
$\mathbf{8}$ & $-6,72$ & 4,9 & $6,6 \pm 0,5$ \\
$\mathbf{9}$ & $-6,58$ & 4,8 & $48,1 \pm 2,6$ \\
$\mathbf{1 0}$ & $-7,95$ & 5,8 & $14,3 \pm 0,5$ \\
$\mathbf{1 1}$ & $-8,14$ & 6,0 & $15,7 \pm 0,8$ \\
$\mathbf{1 2}$ & $-7,8$ & 5,7 & $19,5 \pm 7,8$ \\
$\mathbf{1 3}$ & $-7,59$ & 5,6 & $22,5 \pm 3,5$ \\
$\mathbf{1 4}$ & $-7,79$ & 5,7 & $27,4 \pm 4,4$ \\
$\mathbf{1 5}$ & $-9,07$ & 6,7 & $63,0 \pm 1,7$ \\
$\mathbf{1 6}$ & $-8,27$ & 6,1 & $26,5 \pm 3,5$ \\
\hline
\end{tabular}

complexo mais favorável energeticamente. A partir desses estudos foi possível propor uma explicação para a diferença de atividade entre esses compostos.

Os monoarilfuranos 3, 8 e 9 foram menos ativos tanto em relação aos valores teóricos quanto em relação aos resultados experimentais. A mesma correlação não foi tão evidente em relação aos monoarilfuranos 2, 4 e 6, os quais apresentaram uma certa discrepância entre resultados teóricos e experimentais. Dentre os monoarilfuranos, apenas a benzilamina 5 e a acetamida 9 apresentaram atividade considerável (44 e $48 \%$ de inibição da enzima, respectivamente). A atividade apresentada por $\mathbf{5}$ pode ser justificada em função da possibilidade de interações eletrostáticas entre o grupo $\mathrm{NH}_{3}^{+}$de 5 e os grupos carboxilatos dos resíduos GLU467 e GLU466. Paulino e colaboradores ${ }^{12}$ realizaram estudos de superposição molecular de derivados 5-nitrofurano e 5-nitrotiofeno semi- carbazonas com a TR e GR e enfatizaram a importância da carga positiva para aumentar a especificidade e afinidade dos compostos para a interação com a TR. Em relação à acetamida $\mathbf{9}$, observou-se um maior número de interações com a enzima quando comparada com a acetamida 3 e uma dessas interações envolve o grupo carbonila do aldeído de $\mathbf{9}$ (ligação de hidrogênio com o NH do resíduo de MET400 da TR). Portanto, neste caso, a presença do grupo carbonila teve uma importância efetiva para o aumento da atividade.

A presença da porção semicarbazona e tiossemicarbazona nos compostos 10-13 não ofereceu nenhuma vantagem em relação ao número de interações possíveis com a enzima, quando comparados com a diacetamida $\mathbf{1}$, utilizada como protótipo. $\mathrm{O}$ derivado nitrado 10 apresentou baixa afinidade pela TR (14,3\% de inibição), apesar da sua semelhança estrutural com o 5-nitrofurfural-2-carboxaldeído semicarbazona (Figura 4).

$\mathrm{Na}$ maioria dos casos, os diarilfuranos ocuparam uma região no sítio ativo da enzima correspondente à região ocupada pela cadeia peptídica do substrato natural (tripanotiona) (Figura 5).

Os diarilfuranos 14 e 16 apresentaram, no sítio da TR, uma posição semelhante à da diacetamida $\mathbf{1}$, porém com um menor número de interações, o que refletiu em uma maior energia de encaixe e maior $\mathrm{K} i$ calculado, justificando, assim, sua menor afinidade pela enzima e menor atividade no teste in vitro. Em relação à dibenzilamina 15, observa-se que ocupa uma posição no sítio ativo totalmente diferenciada do restante das moléculas. Foram encontrados três possíveis sítios de interação eletrostática entre a dibenzilamina $\mathbf{1 5}$ com os grupos carboxilato dos resíduos GLU19, GLU113 e ASP117. Além disto, foi observada uma interação do tipo $\pi$ "stacking" do anel furano com o anel indólico do TRP22. Apesar de ela estar em uma posição diferente no sítio ativo, comparada com a tripanotiona, as fortes interações justificam sua afinidade pelo receptor e sua maior atividade, tanto teórica quanto experimental.

A atividade tripanosomicida da dibenzilamina $\mathbf{1 5}$ contra as formas tripomastigotas de T. cruzi também foi avaliada. In vitro, a dibenzilamina 15 foi capaz de eliminar o parasita do sangue murino infectado na dose de $250 \mu \mathrm{g} / \mathrm{mL}$, o que representa uma atividade 20 vezes maior que a da diacetamida 1 (Tabela 2). A maior atividade apresentada por $\mathbf{1 5}$ pode estar relacionada, além da sua maior força de interação com a TR, à sua melhor solubilidade e facilidade de penetração no parasita.

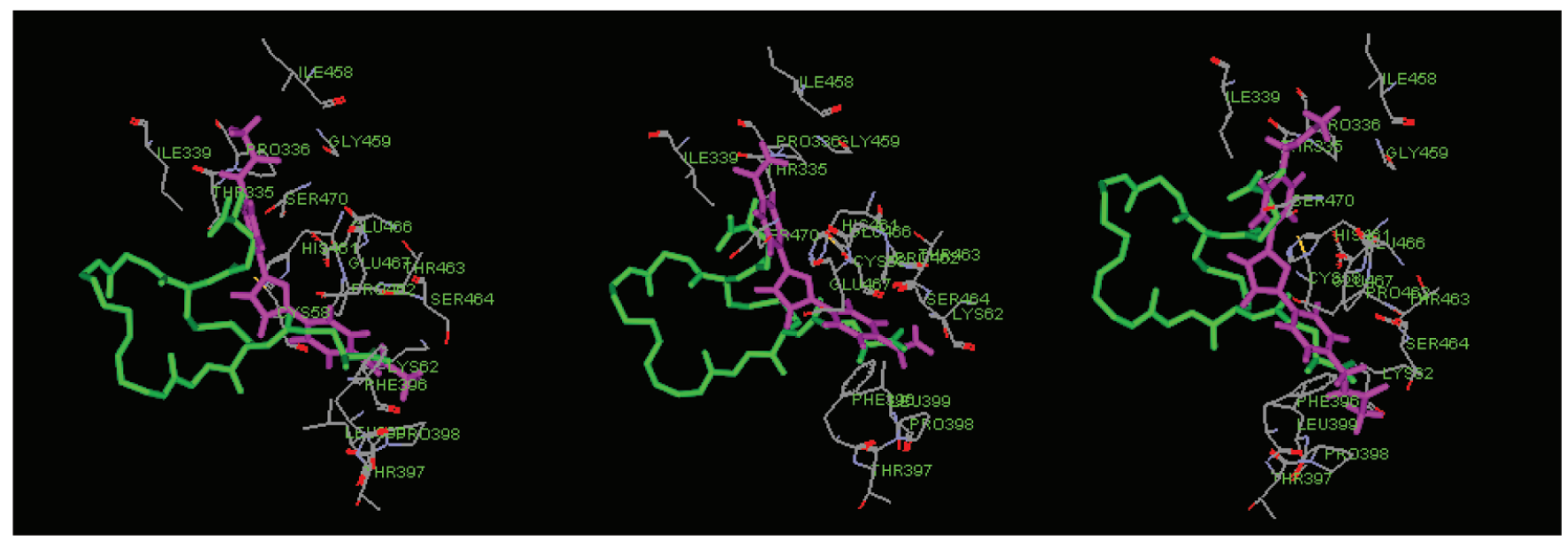

Figura 5. Solução de menor energia encontrada no experimento de superposição molecular da: diacetamida 1 (esquerda); dibenzamida 14 (centro) $e$ dibenzilacetamida 16 (direita) (rosa) com a TR. Em verde, para fins de comparação, a tripanotiona na conformação da estrutura cristalina 
Tabela 2. Comparação entre a atividade tripanosomicida apresentada pela diacetamida $\mathbf{1}$ e dibenzilamina $\mathbf{1 5}$

\begin{tabular}{lcccc}
\hline \multicolumn{5}{c}{ Atividade tripanosomicida $(\% \text { de lise })^{*}$} \\
\hline & Concentração & Concentração & Concentração & Concentração \\
Arilfurano & $250 \mu \mathrm{g} / \mathrm{mL}$ & $125 \mu \mathrm{g} / \mathrm{mL}$ & $62,5 \mu \mathrm{g} / \mathrm{mL}$ & $31,25 \mu \mathrm{g} / \mathrm{mL}$ \\
$\mathbf{1}$ & 5 & - & - & - \\
$\mathbf{1 5}$ & 100 & 97 & 49 & 3,2
\end{tabular}

*Violeta de genciana foi utilizada como controle positivo na concentração de 3,0 $\mu \mathrm{M}\left(\mathrm{IC}_{50}\right)$.

\section{CONCLUSÃO}

Neste trabalho foram sintetizados 15 derivados de mono- e diarilfuranos, sendo 7 inéditos (compostos 4-6, 12-14 e 16). Todas as substâncias sintetizadas foram avaliadas in vitro como potenciais inibidores da TR. Dentre as substâncias testadas, os arilfuranos 5, 9 e $\mathbf{1 5}$ foram os que apresentaram maior capacidade de inibição da enzima (45, 48 e $63 \%$ de inibição, respectivamente). Estudos de superposição molecular utilizando o programa AutoDock 3.0 foram realizados em paralelo, o que nos permitiu propor um modo de interação destas substâncias com a TR. Somente a dibenzilamina $\mathbf{1 5}$ apresentou melhor atividade em relação ao protótipo 1 (62\% de inibição), o que está em concordância com os resultados dos estudos de superposição molecular. A atividade tripanosomicida da dibenzilamina 15 contra as formas tripomastigotas de $T$. cruzi também foi avaliada, sendo 20 vezes mais ativa que o protótipo. A melhor atividade apresentada por $\mathbf{1 5}$ pode estar relacionada tanto a fatores farmacocinéticos (melhor solubilidade) quanto a fatores farmacodinâmicos (maior afinidade pela enzima). Dando prosseguimento a estes estudos, quantidades adicionais da dibenzilamina 15 serão preparadas, visando avaliação de sua atividade in vivo. Nenhuma outra substância apresentou atividade considerável, porém, os resultados obtidos neste trabalho serão de fundamental importância para planejamento de novos inibidores e estudos posteriores.

\section{MATERIAL SUPLEMENTAR}

Os esquemas de síntese e algumas figuras ilustrando as interações entre os mono- e diarilfuranos e a enzima tripanotiona redutase, obtidas a partir dos experimentos de superposição molecular, encontram-se disponíveis em http://quimicanova.sbq.org.br, na forma de arquivo pdf, com acesso gratuito.

\section{AGRADECIMENTOS}

Aos órgãos financiadores CNPq, FAPEMIG e Fiocruz.

\section{REFERÊNCIAS}

1. World Health Organization; http://www.who.int/tdr/diseases, acessada em Agosto 2006.

2. Castro, S. L.; Acta Tropica 1993, 53, 83.

3. Brener, Z.; Andrade, Z. A.; Barral-Netto, M.; Trypanosoma cruzi e Doença de Chagas, $2^{\mathrm{a}}$ ed., Guanabara Koogan: Rio de Janeiro, 2000.

4. Fairlamb, A. H.; Blackburn, P.; Ulrich, P.; Chait, B. T.; Cerami, A.; Science 1985, 227, 1485 .
5. Schmidt, A.; Krauth-Siegel, R. L.; Curr. Top. Med. Chem. 2002, 2, 1239.

6. Chibale, K.; Musonda, C. C.; Curr. Med. Chem. 2003, 10, 1863.

7. Girault, S.; Davioud-Charvet, T. E.; Maes, L.; Dubremets, J. F.; Debreu, M. A.; Landry, V.; Sergheraert, C.; Bioorg. Med. Chem. 2001, 9, 837.

8. Bond, C. S.; Zhang, Y.; Berriman, M.; Cunningham, M. L.; Fairlamb, F. H.; Hunter, W. N.; Structure 1999, 7, 81.

9. Oliveira, R. B.; Vaz, A. B. M.; Alves, R. O.; Liarte, D. B.; Donnici, C. L.; Romanha, A. J.; Zani, C. L.; Mem. Inst. Oswaldo Cruz 2006, 101, 169.

10. Aguirre, G.; Cabrera, E.; Cerecetto, H.; Di Maio, R.; González, M.; Seoane, G.; Duffaut, A.; Denicola, A.; Gil, M. J.; Martínez-Merino, V.; Eur. J. Med. Chem. 2004, 39, 421.

11. Maya, J. D.; Bollo, S.; Nunez-Vergara, L. J.; Squella, J. A.; Repetto, Y.; Morello, A.; Périé, J.; Chauvière, G.; Biochem. Pharmacol. 2003, 65, 999.

12. Paulino, M.; Iribarne, F.; Hansz, M.; Vega, M.; Seoane, G.; Cerecetto, H.; Di Maio, R.; Caracelli, I.; Zukerman-Schpector, J..; Olea, C.; Stoppani, A. O. M.; Berriman, M.; Fairlamb, A. H.; Tapia, O.; J. Mol. Struct. (Theochem) 2002, 584, 95 .

13. Jockers-Scherübl, M. C.; Schirmer, R. H.; Krauth-Siegel, R. L.; Eur. J. Biochem. 1989, 180, 267.

14. Morris, G. M.; Goodsell, D. S.; Halliday, R. S.; Huey, R.; Hart, W. E.; Belew, R. K.; Olson, A. J.; J. Comput. Chem. 1998, 19, 1639.

15. Paizs, C.; Tahtinen, P.; Lundell, K.; Poppe, L.; Irimie, F. D.; Kanerva, L. T.; Tetrahedron: Asymmetry 2003, 14, 1895.

16. King, F. D.; Walton, D. R. M.; Synthesis 1976, 1, 40.

17. Wiesner, J.; Mitsch, A.; Jomaa, H.; Schlitzer, M.; Bioorg. Med. Chem. Lett. 2003, 13, 2159.

18. Costa, P.; Pilli, R.; Pinheiro, S.; Vasconcellos, M.; Substâncias carboniladas e derivados, Artmed Ed. S.A.: Porto Alegre, 2003.

19. Wilson, W. D.; Ratmeyer, L.; Zhao, M.; Strekowski, L.; Boykin, D.; Biochemistry 1993, 32, 4098.

20. Vogel, A. I.; Análise orgânica qualitativa, $3^{\mathrm{a}}$ ed., Ed. Ao Livro Técnico: Rio de Janeiro, 1971, vol. 2.

21. Hashmi, A. S. K.; Choi, J. H.; Bats, J. W.; J. Prakt. Chem. 1999, 341, 342.

22. Mitsch, A.; Wibner, P.; Silber, K.; Haebel, P.; Sattler, I.; Klebe, G.; Schlitzer, M.; Bioorg. Med. Chem. 2004, 12, 4585.

23. White, R. L.; Schwan, T. J.; J. Pharm. Sci. 1976, 65, 135.

24. Holla, B. S.; Malini, K. V.; Rao, B. S.; Sarojini, B. K.; Kumari, N. S.; Eur. J. Med. Chem. 2003, 38, 313.

25. Weiner, S. J.; Kollman, K. A.; Case, D. A.; Singh, U. C.; Ghio, C.; Alagona, G.; Profeta, S. Jr.; Weiner, P. K.; J. Am. Chem. Soc. 1984, 106, 765; Weiner, P.; Kollman, P.; J. Comput. Chem. 1981, 2, 287.

26. Gasteiger, J.; Marsili, M.; Tetrahedron 1980, 36, 3219.

27. Goodford, P. J.; J. Med. Chem. 1985, 28, 849.

28. Höltje, H. D.; Folkers, G.; Molecular modeling basing principles and applications, VCH: Weinheim, 1996.

29. Rondestvedt, C. S.; Org. React. 1976, 24, 225.

30. Beraldo, H.; Quim. Nova 2004, 27, 461.

31. Cerecetto, H.; Di Maio, R.; González, M.; Risso, M.; Sagrera, G.; Seoane, G.; Denicola, A.; Peluffo, G.; Quijano, C.; Stoppani, A. O. M.; Paulino, M.; Olea-Azar, C.; Basombrío, M. A.; Eur. J. Med. Chem. 2000, 35, 343.

32. Holloway, M. K. Em 3D QSAR in Drug Design - Ligand-Protein Interactions and Molecular Similarity; Kubiny, H.; Folkers, G.; Martin, Y., eds.; Kluwer Academic Publishers: Dordrecht, 1998, vol. 2, p. 63-84. 